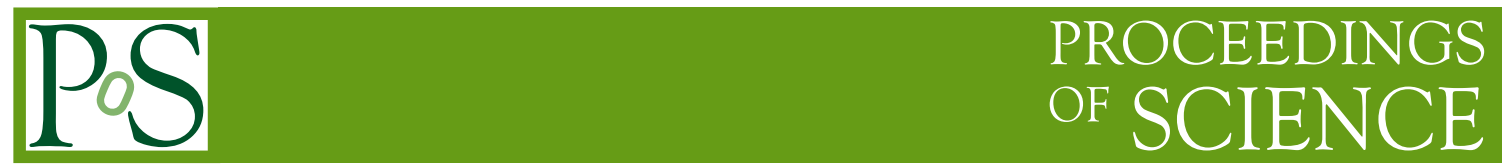

\title{
The NA62 Experiment at CERN
}

\section{Giuseppe Ruggiero*}

CERN, Geneve

E-mail: giuseppe.ruggiero@cern.ch

The NA62 experiment, which aims to measure the branching ratio of the very rare kaon decay $K^{+} \rightarrow \pi^{+} v \bar{v}$ at the CERN SPS, is described. The proposed experiment aims to collect some 100 $K^{+} \rightarrow \pi^{+} v \bar{v}$ events with a $10 \%$ of background. The status and the future perspectives for the experiment are discussed.

The Xth Nicola Cabibbo International Conference on Heavy Quarks and Leptons, October 11-15, 2010 Frascati (Rome) Italy

\footnotetext{
* Speaker.
} 


\section{Introduction}

Among the many rare flavour changing neutral current $K$ and $B$ decays, the rare decays $K \rightarrow$ $\pi \nu \bar{v}$ play a key role in search for new physics through underlying mechanisms of flavour mixing. The Standard Model (SM) branching ratio can be computed to an exceptionally high degree of precision: the $O\left(G_{F}^{2}\right)$ electroweak amplitudes exhibit a power-like GIM mechanism; the top-quark loops largely dominate the matrix element; the sub-leading charm-quark contributions have been computed at NNLO order [1]; the hadronic matrix element can be extracted from the branching ratio of the $K^{+} \rightarrow \pi^{0} e^{+} v$ decay, well known experimentally [2]. The SM prediction for the $K^{+} \rightarrow$ $\pi^{+} v \bar{v}$ channel is $(8.5 \pm 0.7) \times 10^{-11}$ [3]. The error comes mainly from the uncertainty on the CKM matrix elements, while the irreducible theoretical uncertainty amounts to less than $2 \%$. The extreme theoretical cleanness of these decays remains also in new physics scenarios like Minimal Flavour Violation (MFV) [4] or non-MFV models [5] and even not large deviations from the SM value (for example around 20\%) can be considered signals of new physics.

The decay $K^{+} \rightarrow \pi^{+} v \bar{v}$ has been observed by the stopping kaon experiments E787 and E949 at the Brookhaven National Laboratory and the measured branching ratio is $1.73_{-1.05}^{+1.15} \times 10^{-10}$ [6]. However only a measurement of the branching ratio with at least $10 \%$ accuracy can be a significant test of new physics. This is the main goal of the NA62 experiment at CERN-SPS [7, 8]. It aims to collect some $100 \mathrm{~K}^{+} \rightarrow \pi^{+} v \bar{v}$ events in about two years of data taking, keeping a background contamination around $10 \%$.

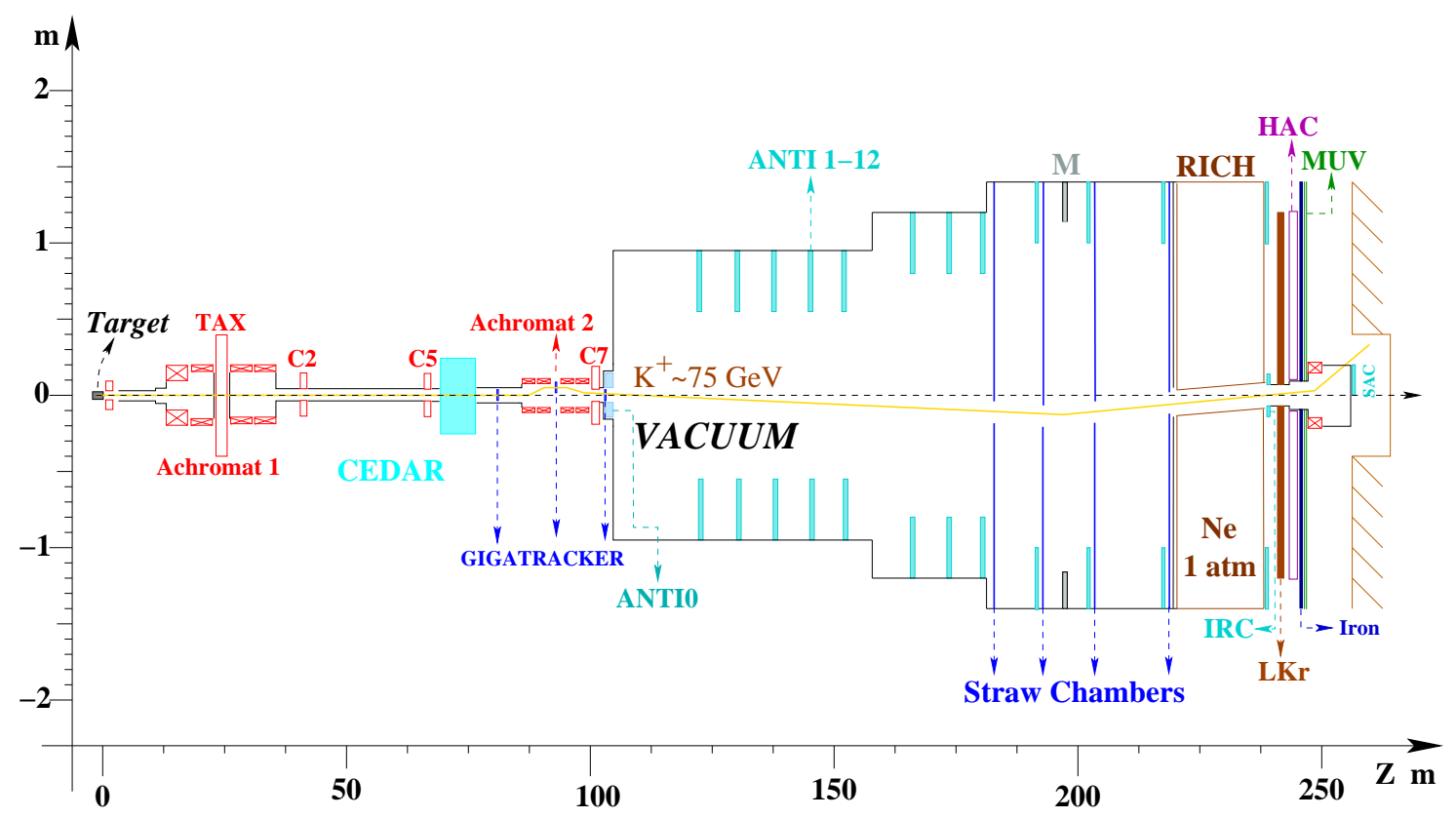

Figure 1: Layout of the experiment.

\section{The NA62 Experiment}

Figure 1 shows the layout of the NA62 experiment. The requirement of 100 events needs to $10 \%$ signal acceptance and at least to $10^{13} \mathrm{~K}^{+}$decays. The required signal to background ratio 
demands a background suppression factor of at least $10^{13}$. A high energy kaon beam and a decayin-flight technique are the principles of the experiment.

The experiment will be housed in the CERN North Area High Intensity Facility (NAHIF) where the NA48 [9] was located and it will use the same SPS extraction line and target of NA48. A new high acceptance beam line will deliver a 50 times more intense secondary hadron beam of positive charge of $75 \mathrm{GeV} / \mathrm{c}$ momentum $( \pm 1 \%)$, corresponding to an average rate of about 800 $\mathrm{MHz}$ integrated over an area of $14 \mathrm{~cm}^{2}$. The beam is positron free and is composed by $6 \%$ of $\mathrm{K}^{+}$. A system of subdetectors placed about $170 \mathrm{~m}$ downstream to the target provides the detection of the $K^{+}$decay products. The average integrated rate on the detectors downstream is about $10 \mathrm{MHz}$, mainly due to the kaon decays and accidental muons. This beam line provides $5 \times 10^{12} K^{+}$decays, assuming $60 \mathrm{~m}$ decay region and 100 days of run at $60 \%$ of efficiency, which is a very realistic estimate based on the decennial NA48 experience at the SPS.

The key points of NA62 are: an accurate kinematic reconstruction to disentangle the signal; a precise timing to associate the $\pi^{+}$with its $K^{+}$parent; a system of efficient vetoes to reject events with $\gamma$ and $\mu$; a particle identification system to identify the kaons in the charged beam and to distinguish $\pi^{+}$from $\mu^{+}$and $e^{+}$in the final state.

The $R \& D$ of the experiment finished at the end of 2010. The construction of the apparatus is already started and the beginning of the data taking is foreseen in 2013.

\section{Kinematic Rejection}

The kinematics of $K^{+} \rightarrow \pi^{+} v \bar{v}$ decay allows the signal definition. The main variable in use is the squared missing mass, $m_{\text {miss }}^{2}$, defined as the square of the difference of the 4-momenta of the $K^{+}$ and of the track downstream in the $\pi^{+}$hypothesis. This variable separates the signal from more than
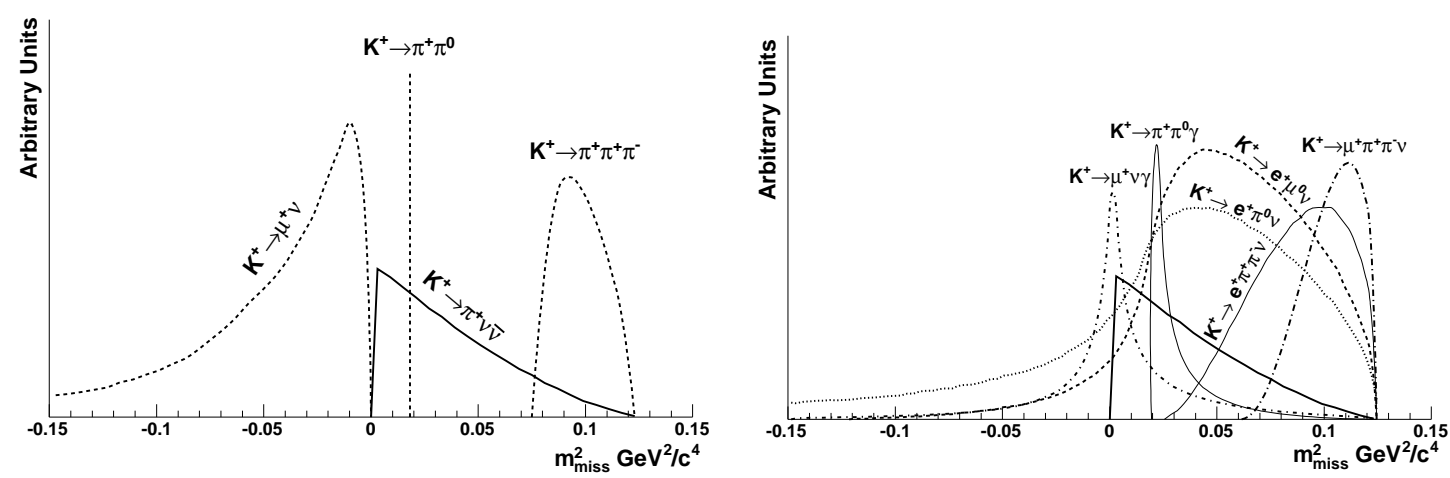

Figure 2: Squared missing mass for the main Kaon decay modes: with kinematic thresholds (left); without kinematic thresholds (right).

$90 \%$ of the background coming from other $K^{+}$decays (Figure 2 left). The $K^{+} \rightarrow \pi^{+} \pi^{0}$ peak divides two regions of $m_{\text {miss }}^{2}$ containing a minimal amount of background. Non signal events enter here from decays with kinematic thresholds because of kinematic resolution effects (e.g. $K^{+} \rightarrow \pi^{+} \pi^{0}$, $K^{+} \rightarrow \mu^{+} v_{\mu}$ ), or from decays without kinematic thresholds, as the radiative ones (Figure 2 right). Against the first source of background low mass and high precision detectors placed in vacuum 
are mandatory for tracking. A beam tracker along the beam line and a spectrometer downstream to the decay region accomplish this task. The very high rate in the beam detector requires also to associate the incoming $K^{+}$to the downstream $\pi^{+}$by means of tight spatial and time coincidences. The beam tracker itself and a RICH downstream provide the timing of the experiment. Notably the beam tracker must reconstruct the tracks with at least $200 \mathrm{ps}$ time resolution.

The designed beam spectrometer (Gigatracker) consists of $3 \mathrm{Si}$ pixel stations matching the beam size. A Si sensor $200 \mu \mathrm{m}$ thick and a readout chip $100 \mu \mathrm{m}$ thick bump bonded on the sensor form one pixel. Beam tests on prototypes performed at CERN in 2010 showed a time resolution in agreement with the requested one.

Four chambers made by straw tubes and placed in the same vacuum of the decay region form the downstream pion spectrometer. Four planes of tubes oriented along the $x, y$ and $\pm 45^{\circ}$ directions form each chamber. They provide the measurement of the coordinates of the impact point of the tracks. Regions free of tubes in each plane create an octagonal hole around the beam axis in the chambers where the beam of particles passes through. The same NA48 dipole magnet, placed after the second chamber, allows the momentum analysis. The chambers are displaced in the bending plane according to the $75 \mathrm{GeV} / \mathrm{c}$ positive beam path, mainly to veto additional high momentum charged particles. Full-length plane prototypes operating in vacuum have been tested at CERN using hadron beams in 2007, 2009 and 2010. The tests showed that the single coordinate can be reconstructed with a resolution better than $100 \mu \mathrm{m}$.

A Geant4 [10] Monte Carlo simulation of the above detectors predicted a resolution on $\mathrm{K}^{+}$ momentum of about $0.2 \%$, on $K^{+}$direction of about $16 \mu \mathrm{rad}$, on $\pi^{+}$momentum of about $0.3 \% \oplus$ $0.007 \% \times P_{\text {track }}(\mathrm{GeV} / \mathrm{c})$ and on $\pi^{+}$direction between 15 and $45 \mu \mathrm{rad}$, depending on the momentum. The expected kinematic rejection power against $K^{+} \rightarrow \pi^{+} \pi^{0}$ and $K^{+} \rightarrow \mu^{+} v_{\mu}$ decays is of about $10^{4}$ and $10^{5}$, respectively.

\section{Photon and Muon Vetoes}

An additional rejection factor, largely independent from the kinematics, comes from $\gamma$ and $\mu$ detection.

The $K^{+} \rightarrow \pi^{+} \pi^{0}$ suppression drove the design of the photon veto system, giving a requirement of about $10^{-8}$ inefficiency for $\pi^{0}$ rejection. The photon veto system should guarantee a good level of $K^{+} \rightarrow \pi^{+} \pi^{0}$ suppression already online, in order to reduce the rate for data acquisition. The detectors designed for these goals are: a system of calorimeters (LAV) covering the angle region between 8.5 and $50 \mathrm{mrad}$; an electromagnetic calorimeter between 1 and $8.5 \mathrm{mrad}$ and small angle calorimeters covering the region below $1 \mathrm{mrad}$. The key points are: an offline sub-nanosecond time resolution of all the devices and online for the calorimeter in the 1-8.5 mrad region; a cut at $35 \mathrm{GeV} / \mathrm{c}$ on the maximum $\pi^{+}$momentum offline to deal with $\pi^{0}$ of at least $40 \mathrm{GeV} / \mathrm{c}$; a detection inefficiency below $10^{-5}$ for $\gamma$ 's in the $1-8.5 \mathrm{mrad}$ region above $10 \mathrm{GeV}$ and, anyhow, within $10^{-3}$ down to $1 \mathrm{GeV}$.

The electromagnetic liquid Kripton calorimeter of NA48 (LKr) will be reused to veto $\gamma$ 's in the 1-8.5 mrad region. Measurements using $K^{+} \rightarrow \pi^{+} \pi^{0}$ selected on NA48 data have demonstrated the capability of the $\mathrm{LKr}$ to reach the required veto performance. The sub-nanosecond time resolution 
online of the LKr, already obtained by the NA48 experiment, makes this detector essential for the trigger.

Twelve rings surrounding the NA62 decay and detector regions and placed in vacuum form the LAV system. The positions of the rings along the experiment guarantee the required angular coverage. The lead glass counters from the electromagnetic calorimeter of the LEP experiment OPAL [11] are the building blocks of the rings. They guarantee a level of inefficiency around $10^{-4}$ down to $0.5 \mathrm{GeV}$ photons, as measured in test beams performed at the Dafne Beam Test facility in Frascati, using positrons [8]. The first two rings have been mounted and successfully tested at CERN on the hadron beam line in 2009 and 2010. The data showed a sub-nanosecond time resolution.

The suppression of the $K^{+} \rightarrow \mu^{+} v_{\mu}$ decay drove the design of the muon veto system. The maximum tolerable inefficiency for the muon rejection turned out to be in the range of $10^{-3}$ online and $10^{-5}$ offline. Given the integrated rate on the muon veto system of about $10 \mathrm{MHz}$, a subnanosecond time resolution online is also a crucial request for the muon veto detectors.

The muon detection system will make use of an upgraded version of the hadronic calorimeter of NA48 (MUV), for the offline rejection and of a plane of fast $20 \times 20 \mathrm{~cm}^{2}$ pad-scintillators (MUV3) placed at the end of the apparatus after an iron wall, for the trigger. Test beams, performed in 2010 on a MUV3 prototype, showed the possibility to reach an online time resolution around 500-600 ps, thanks to the use of two photomultipliers (PM) without light-guide to readout one single pad. The two PMs, in particular, give enough redundancy to solve the signal ambiguity due to the Cerenkov light eventually produced by particles impinging on the photocathode.

Monte Carlo simulations predicted an inefficiency of muon detection around $10^{-5}$, achievable by exploiting the electromagnetic and hadronic shower separation capability of the hadronic calorimeter together with the $\mathrm{LKr}$ detector.

\section{Particle Identification}

The kinematic rejection and the muon veto alone are still unable to provide enough suppression of backgrounds like $K^{+} \rightarrow \mu^{+} v_{\mu}(\gamma)$. An identification device for the $K^{+}$decay products, different from the calorimeters, must provide the missing $10^{2}$ factor in the rejection of this background.

A RICH has been designed to fulfill such a goal: it should separate $\pi^{+}$from $\mu^{+}$with inefficiency below $1 \%$. It must also provide the timing of the event with a resolution below $100 \mathrm{ps}$ and it should be used as a trigger for 1-track events. A vessel $17 \mathrm{~m}$ long placed after the pion spectrometer and filled with $\mathrm{Ne}$ at atmospheric pressure forms the detector. The vessel has a cylindrical shape around a $17 \mathrm{~cm}$ diameter beam tube used to let the undecayed particles to pass through in vacuum. A mosaic of mirrors at the end, having $17 \mathrm{~m}$ focal length, reflects the Cerenkov light towards two arrays of about 1000 phototubes each, placed on both the sides of the vessel at the entrance window. Hamamatsu phototubes of $1.8 \mathrm{~cm}$ diameter guarantee a quantum efficiency and time performances which fit the requirements. Test beams, performed at CERN SPS in 2007 and 2009 on full-length prototypes equipped with 400 photomultipliers, showed that a $\pi^{+} / \mu^{+}$separation with the requested purity can be reached in the $15-35 \mathrm{GeV} / \mathrm{c}$ momentum range, for a time resolution around $70 \mathrm{ps}[12,13]$. As a by-product the $\mathrm{RICH}$, together with the $\mathrm{LKr}$, is also suitable for $\pi / \mathrm{e}^{ \pm}$separation with a mis-identification probability below $10^{-3}$. 
A particle identification detector on the beam line is also mandatory against accidentals. Dangerous accidental events come from the interactions of the beam particles mainly just before entering in the decay region (e.g. interactions with the last station of the Gigatracker or with the residual gas in the vacuum). Since $94 \%$ of the particles are protons and $\pi^{+}$, a Cerenkov Threshold Counter (CEDAR) placed on the beam line, which positively recognizes the kaons, allows the rejection of the most part of the accidentals. The CEDAR is an existing detector built at CERN in 70's [14] and a program of refurbishing both the radiating material and the detection part already started within the NA62 collaboration.

\section{Trigger and Data Acquisition}

The trigger should reduce the online $10 \mathrm{MHz}$ rate per detector to $10 \mathrm{kHz}$ in order to make the data acquisition feasible, given the event size from each detector and the data bandwidth as principal hardware constraints. A three-level trigger system should accomplish this task. All the detectors, but the Gigatracker and the LKr, send asynchronously the digitized signal to a custommade board which hosts the CERN HPTDC's [15]. The TDC's provide the leading and trailing time of the incoming signal and send it to an upgraded version of the LHC TELL1 board [16]. This module processes the timing information, builds the trigger primitives of the corresponding detector and sends them to a L0 central processor (LOTS). The LOTS elaborates these inputs with a latency time in the range of some ms, issues a trigger decision and sends it back to the TELL1-like boards. In case of positive decision the boards allows the readout of the data to dedicated detector PC's. The LKr uses FADC's instead of TDC's. It also makes use of two independent systems for trigger and readout. The LKr, the MUV3 and the RICH mainly contribute to the L0 trigger decision, providing a suppression factor greater than 10.

The Level 1 and 2 trigger are based on PC's. The detector PC's process the $1 \mathrm{MHz}$ data rate which passes the L0 trigger and define more complete information in order to build a L1 trigger decision. The filtered data are sent further to a gigabit Ethernet switch and then to a PC farm which provides global event information for the ultimate trigger word. The raw data are finally assembled by dedicated event-building PC's and transferred for storing with a maximum speed of event logging on tape in the range of $100 \mathrm{MB} / \mathrm{s}$.

The online sub-nanosecond time resolution of the main veto detectors and the RICH are crucial features in order to guarantee a signal efficiency of the trigger greater than $95 \%$. The minimization of the custom-made hardware part allows an offline reproducibility of the trigger conditions for monitoring purposes. The stringent offline requirements on veto efficiencies need for no zero suppression of the candidate events: as a consequence a Gbit/s data bandwidth capability is crucial for the whole trigger and readout chain.

\section{Sensitivity}

A preliminary analysis has been done using Geant3 [17], Geant4 and Fluka [19] based simulations. The total acceptance is about $14.4 \%$, showing that the target of $10 \%$ of signal acceptance is safely achievable even taking into account additional losses occurring in a real data taking. The use of the RICH constrains the accepted pion tracks within the $(15,35) \mathrm{GeV} / \mathrm{c}$ momentum range. 
The higher cut is an important loss of signal acceptance, but assures that events like $K^{+} \rightarrow \pi^{+} \pi^{0}$ deposit at least $40 \mathrm{GeV}$ of electromagnetic energy, making their rejection easier.

Many sources of background have been considered and just a simple counting of signal and background events in the signal regions indicates that the $10 \%$ background appears to be within reach.

\section{Conclusions}

The ultra-rare $K \rightarrow \pi v v$ decay is a unique environment where to search for new physics. The NA62 experiment at CERN-SPS proposes to follow this road by collecting $O(100)$ events of the $K^{+} \rightarrow \pi^{+} v \bar{v}$ decay. The experiment has been approved and funded. After three years of successful $\mathrm{R} \& \mathrm{D}$ program, the NA62 experiment is now under construction.

\section{References}

[1] A. J. Buras, M. Gorbahn, U. Haisch and U. Nierste, JHEP 0611, 002 (2006) HEP-PH 0603079.

[2] C. Amsler et al., Phys. Lett. B667, 1 (2008).

[3] J. Brod and M. Gorbahn, Phys. Rev. D 78034006 (2008)

[4] G. Isidori, F. Mescia, P. Paradisi, C. Smith, S. Trine, HEP-PH 0604074. JHEP 0608, 064 (2006).

[5] M. Blanke, A.J. Buras, A. Poschenrieder, S. Recksiegel, C. Tarantino, S. Uhlig and A. Weiler, HEP-PH 0610298. JHEP 0701, 066 (2007).

[6] A. V. Artamonov et al. [BNL-E949 Collaboration], Phys. Rev. D 79, 092004 (2009)

[7] G. Anelli et al., CERN-SPSC-2005-013, SPSC-P-326.

[8] NA62 Collaboration, CERN-SPSC-2007-035, SPSC-M-760.

[9] V. Fanti et al. [NA48 Collaboration], Nucl. Instrum. Meth. A 574, 433 (2007).

[10] S. Agostinelli et al. [GEANT4], Nucl. Instrum. Meth. A 506, 250 (2003).

[11] K. Ahmet et al. [OPAL Collaboration], Nucl. Instrum. Meth. A 305, 275 (1991).

[12] G. Anzivino et al., Nucl. Instrum. Meth. A 593, 314 (2008).

[13] B. Angelucci et al., Nucl. Instrum. Meth. A 621, 205 (2010).

[14] C. Bovet, et al., The CEDAR Counters for Particle Identification in the SPS Secondary Beams, CERN Report: CERN 82-13 (1982).

[15] J. Christiansen, "HPTDC High Performance Time to Digital Converter", CERN, Geneva, 2004, Version 2.2 for HPTDC version 1.3.

[16] G. Haefeli, A. Bay, A. Gong, H. Gong, M. Muecke, N. Neufeld and O. Schneider, Nucl. Instrum. Meth. A 560, 494 (2006).

[17] CERN Program Library Long Writeup, W5013 (1993).

[18] J. Allison et al., IEEE Trans. Nucl. Sci. 53, 270 (2006). IETNA,53,270.

[19] A. Ferrari, P.R. Sala, A. Fasso and J. Ranft, CERN-2005-010. 